\title{
Bouncing droplets on nonsuperhydrophobic surfaces
}

\author{
Longquan Chen and Zhigang $\mathrm{Li}$ \\ Department of Mechanical Engineering, The Hong Kong University of Science and Technology, Clear Water Bay, \\ Kowloon, Hong Kong
}

(Received 16 March 2010; revised manuscript received 5 May 2010; published 14 July 2010)

\begin{abstract}
It is known that impinging droplets can rebound off superhydrophobic while tend to wet hydrophilic and hydrophobic surfaces. In this work, we show that water droplets can bounce off nonsuperhydrophobic soft surfaces between lower and upper impact velocity limits. The cause for the rebound of the droplet is hypothesized as the formation of an air film at the interface due to the deformability of the surface when the impact velocity is larger than the lower limit. At large impact velocity, an air bubble is entrapped from the cavity formed during the spreading of the droplet on the surface. This air bubble coalesces with and releases the air film beneath the droplet when the impact velocity is larger than the upper limit, beyond which the surface becomes wetting and the rebound of droplets is not observed.
\end{abstract}

DOI: 10.1103/PhysRevE.82.016308

PACS number(s): 47.55.D-, 68.08.Bc

Inspired by rolling water droplets on lotus leaves and motivated by the potential applications [1-3], droplet-surface interactions have attracted great interest recently. Depending on the surface energy and roughness, a surface can be hydrophilic, hydrophobic, or superhydrophobic. On hydrophilic surfaces, a water droplet tends to wet the surface and exhibits small static contact angles. For hydrophobic surfaces, the contact angle is usually larger than $90^{\circ}$. Superhydrophobic surfaces are characterized by a contact angle larger than $150^{\circ}$ and a low contact angle hysteresis [4]. Lotus leaves are a representative biosurface showing superhydrophobicity. Mimicking lotus leaves by fabricating superhydrophobic surfaces has been the objective of many attempts [5-11]. Statically, most of the artificial surfaces demonstrate similar wetting properties to lotus leaves and have a static contact angle larger than $150^{\circ}$. Dynamically, however, they may not really behave like a lotus leaf when droplets impact on them. Therefore, the dynamic impingement of liquid droplets has also been employed to characterize the wettability of various surfaces [3,11-17].

The laboratory-fabricated superhydrophobic surfaces are usually made from low-energy materials and have microstructured and/or nanostructured roughness [5-11]. When a droplet impacts on such surfaces, the air trapped by the droplet and rough structures of the surface can offer a considerable capillary pressure to help the droplet rebound off the surface [3,11-17]. However, when a droplet impinges on a hydrophilic or hydrophobic surface, the droplet tends to wet the surface through either spreading on or sticking onto the surface $[13,14,16-18]$. This is especially true for rigid surfaces. In the literature, most of the bouncing droplets were observed on superhydrophobic surfaces. Little work has been reported about the droplet rebound on hydrophilic or hydrophobic surfaces $[19,20]$.

In this work, we report the dynamics of millimetric water droplets impinging on soft surfaces, whose wettablility can be tuned from hydrophilic to hydrophobic with an apparent contact angle (ACA) varying from about $13^{\circ}$ to $110^{\circ}$. It is found that these statically nonsuperhydrophobic surfaces dynamically exhibit superhydrophobicity. The droplets can rebound off the surfaces in a range of impact velocities. The bounce of the droplets is caused by the formation of an air film at the interface, which greatly reduces the surface energy. The lower and upper limits of the impact velocity for the rebound are measured and discussed.

The soft surfaces are made from polydimethylsiloxane. The deformability of the surfaces can be varied by changing the cross-link density. The first surface was fabricated by coating a $300-\mu \mathrm{m}$-thick film of pure silicone elastomer on a glass wafer. The substrate was then introduced into an oven and heated up at $343 \mathrm{~K}$ for $15 \mathrm{~min}$ to remove the air bubbles in the film. After the substrate was cooled down in air, a $7 \mu$ water droplet of radius $R=1.2 \mathrm{~mm}$ was brought in contact with the substrate. At the very beginning, the shape of the droplet was quite spherical with ACA $\theta=132^{\circ}$, which deviates from the equilibrium contact angle $\theta_{0}$, as shown in Fig. 1(a). Due to the nonzero net horizontal force $\gamma_{\mathrm{LV}}\left(\cos \theta-\cos \theta_{0}\right)$ caused by the surface tensions, $\gamma_{\mathrm{LV}}, \gamma_{\mathrm{LS}}$, and $\gamma_{\mathrm{SV}}$, at the liquid-solid-vapor interfaces [Fig. 1(b)], the droplet started to spread until it reached the final equilibrium state. For droplets spreading on a soft surface, the vertical surface tension $\gamma_{\mathrm{LV}} \sin \theta$ deforms the surface and a "wetting bridge" with a height of $d=\gamma_{\mathrm{LV}} \sin \theta /|G|$ is formed [21,22] [Fig. 1(b)], where $G$ is the complex shear modulus of the

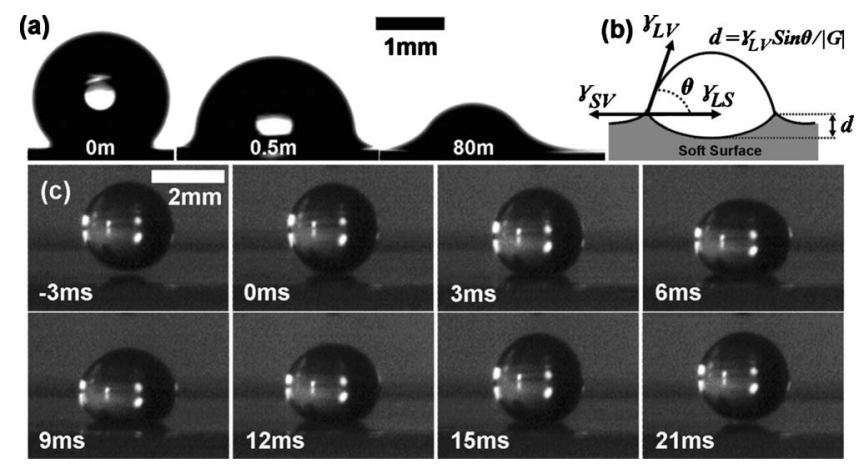

FIG. 1. (a) The spreading of a $7 \mu$ water droplet on the silicone elastomer surface. (b) Diagram of surface tensions, $\gamma_{\mathrm{LV}}, \gamma_{\mathrm{LS}}$, and $\gamma_{\mathrm{SV}}$ at the liquid-solid-vapor interfaces. The vertical surface tension deforms the soft surface and causes a wetting bridge of height $d$. (c) Snapshots of a water droplet impinging on the surface in (a) with impact velocity $V=0.037 \mathrm{~m} / \mathrm{s}$. 
surface. This wetting bridge dissipates the energy of the moving contact line and slows down the spreading of the droplets. In Fig. 1(a), the spreading of contact line stopped after about $80 \mathrm{~min}$ and the final contact angle was measured to be $\theta_{0}=13.4^{\circ}$. The time required for the droplet to reach the final steady state depends on the viscoelastic property of the surface. The magnitude of the shear modulus $|G|$, which was measured by a rheometer (Haake RS1, the modulus was measured at a shear rate of $400 \mathrm{~s}^{-1}$ for all the surfaces in this work), was $1.26 \mathrm{kPa}$. Therefore, the surface was quite "soft" and hydrophilic.

However, when a droplet of the same size was freed slightly above the surface from a height of $0.07 \mathrm{~mm}$ (the corresponding impact velocity $V=0.037 \mathrm{~m} / \mathrm{s}$ ), instead of wetting the surface, the droplet bounced off the surface, as shown in Fig. 1(c). The rebound of the droplet was observed when the impact height was increased up to $11.26 \mathrm{~mm}$, for which the impact velocity was $0.47 \mathrm{~m} / \mathrm{s}$. When the impact height was larger than this value, the droplet started to wet the surface and the rebound of the droplet was not found.

The rebound of the droplet on the hydrophilic surface is surprising because it was assumed only possible on superhydrophobic surfaces. The dynamics of a droplet impinging on a surface is determined by the competition between the wetting and antiwetting pressures [3]. There are two wetting pressures, i.e., water hammer pressure $P_{W H}=\rho_{W} C_{W} V$ (rigid surface) and dynamic pressure $P_{D}=\rho_{W} V^{2} / 2$, where $\rho_{W}$ is the water density and $C_{W}$ is the speed of sound in water. $P_{W H}$ is generated due to the shock wave built up by the compression of droplet when it gets in contact with the surface [23]. If the surface is soft, the surface will deform and the actual water hammer pressure $P_{W H}^{\prime}$ can be estimated by [24]

$$
P_{W H}^{\prime}=\rho_{W} C_{W} V /\left[1+\left(\rho_{W} C_{W} / \rho_{S} C_{S}\right)\right],
$$

where the subscript " $S$ " denotes surface. In Eq. (1), the surface density $\rho_{S}$ and the speed of sound in the surface, $C_{S}$, are related to the surface property. They might account for the dependence of $P_{W H}^{\prime}$ on the deformability of the surface. The antiwetting pressure is the capillary pressure $P_{C}$ which is caused by the air trapped by the surface roughness. For a droplet to rebound off a surface, the nonwetting condition $P_{C}>P_{W H}>P_{D}$ has to be satisfied [3].

For superhydrophobic surfaces, the microstructures and/or nanostructures on the surface can generate a relatively large $P_{C}$ to meet the nonwetting requirements. However, for the surface in Fig. 1(a), the root-mean-square roughness, characterized by an interferometer (Veeco Wyko NT1100), is less than $1.5 \mathrm{~nm}$. The antiwetting pressure $P_{C}$ for such a flat surface is less than $1 \mathrm{~Pa}$ [17], which is much smaller than $P_{W H}^{\prime}=22 \mathrm{kPa}\left(\rho_{S}=970 \mathrm{~kg} / \mathrm{m}^{3}\right.$ and $\left.C_{S} \approx 1020 \mathrm{~m} / \mathrm{s}\right)$. Therefore, the nonwetting condition cannot be used to explain the rebound of the droplet in Fig. 1(c).

Actually, when a droplet approaches a rigid surface, the air in the gap between the droplet and surface is squeezed out and the air pressure is increased. When the droplet is very close to the surface, the high air pressure in the gap deforms the droplet and the air in the dent is entrapped at the point of contact [25-27], as shown in Fig. 2(a). The surface tension will force the air entrapped to form a bubble, which will
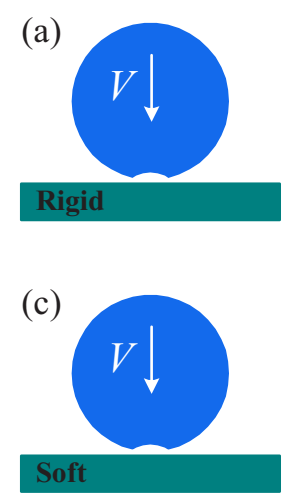

(b)

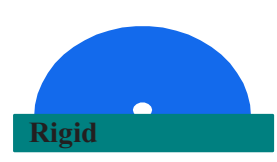

(d)

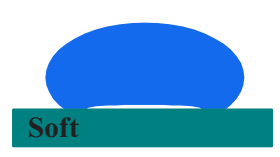

FIG. 2. (Color online) The formation of air bubble and film when a droplet impacts on rigid and soft surfaces. (a) The high air pressure in the gap deforms the droplet and the air in the dent is to be entrapped. (b) The entrapped air forms a bubble on rigid surfaces. (c) The deformation of the droplet is similar to (a) (the deformation of the soft surface is not shown). (d) The entrapped air forms a thin film and the surface becomes superhydrophobic.

basically remain its shape when the droplet spreads on the surface [25] [Fig. 2(b)]. However, if the surface is soft, the dynamics of the air entrapped can be very different after the contact. The surface in Fig. 1 is viscoelastic and shows shear thinning behavior (apparent viscosity decreases with increasing shear rate). The viscosity of the surface is plotted as a function of shear rate in Fig. 3. For such a surface, the air entrapped at the moment of contact can generate a large shear force under $P_{W H}$. This shear force greatly reduces the viscosity of and easily deforms the surface. The entrapped air then forms an air film at the interface instead of an air bubble, as depicted in Figs. 2(c) and 2(d). The difference between the air bubble and film means a lot for impinging droplets. The area of the bubble-surface interface is very small compared with the wetted area, and the bubble can barely affect the droplet-surface interaction. However, the air film may occupy a large fraction of the wetted area, considerably reduce the surface energy, and make the surface superhydrophobic. In this case, a droplet will tend to rebound off the surface after impinging on it. This explains why the droplet bounced off the hydrophilic surface in Fig. 1(c).

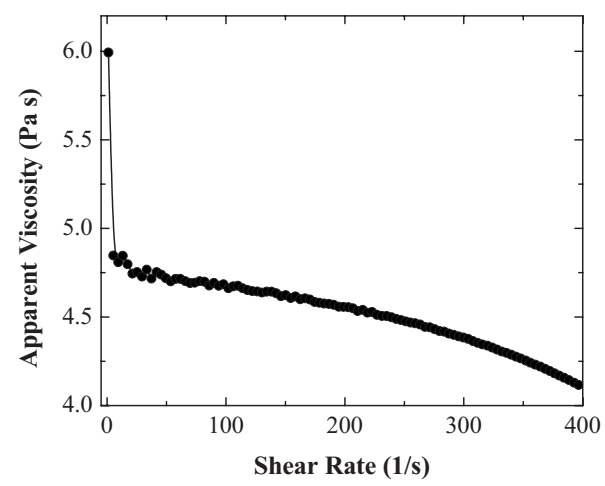

FIG. 3. Apparent viscosity of the surface in Fig. 1 as a function of shear rate. 


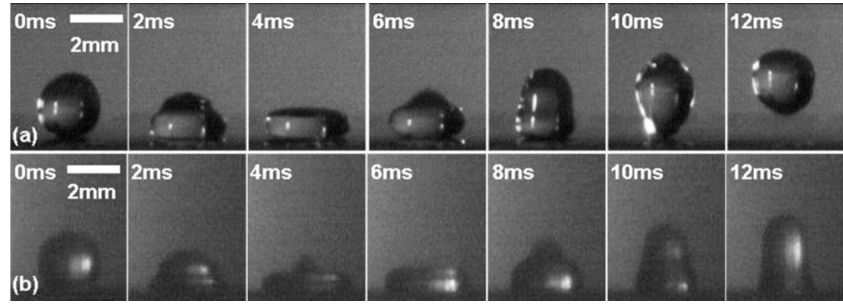

FIG. 4. Snapshots of droplets impacting on the surface in Fig. 1 with $V=0.3 \mathrm{~m} / \mathrm{s}$ under different air pressures. (a) $P_{\text {air }}=1$ atm and (b) $P_{\text {air }}=0.53$ atm.

It is difficult to show the dynamics of the entrapped air during the impact of the droplet, and the above explanation is just our hypothesis. Actually, the previous work about the impingement of silicone oil droplets on the same fluid has demonstrated the existence of the air film, which leads to the rebound of the droplets [20]. Nevertheless, to support the hypothesis, we performed droplet impact experiments by changing the ambient air pressure. Figure 4(a) shows the sequential images of a droplet impinging on the surface in Fig. 1 with an impact velocity $V=0.3 \mathrm{~m} / \mathrm{s}$ at atmospheric pressure $(1 \mathrm{~atm})$, where the droplet bounced off the surface. However, when the same experiment was conducted in a vacuum chamber and the pressure of the camber was reduced to $0.53 \mathrm{~atm}$, the droplet failed to bounce off the surface, as shown in Fig. 4(b). This indirectly confirms that the air film plays important roles in the rebound of the droplet. It is noted that both shear thinning property of the surface viscosity and air penetration in the surface may affect the dynamics of the droplet. The experiment in Fig. 4(b), to some extent, can account for the viscosity effects because the air pressure between the droplet and surface decreases when the ambient air pressure is reduced, which will change the viscosity of the surface. The air penetration in the surface, however, may not play an important role because it can prevent the droplet from bouncing off the surface and this can be excluded based on the observations in Fig. 1(c).

Since the formation of the air film is caused by the water hammer pressure $P_{W H}$ and deformation of the surface, there should exist a lower limit for the impact velocity, below which $P_{W H}$ is insufficient to deform the surface and the droplet will not bounce back. This lower limit should depend on the deformability of the surface. To confirm this, we further fabricated three surfaces with different viscoelasticities by adjusting the cross-link density. The shear moduli and ACAs are (a) $|G|=4.62 \mathrm{kPa}\left(\mathrm{ACA}=40.74^{\circ}\right)$, (b) $|G|=7.27 \mathrm{kPa}$ $\left(\mathrm{ACA}=102.8^{\circ}\right)$, and $(\mathrm{c})|G|=11.83 \mathrm{kPa}\left(\mathrm{ACA}=111.2^{\circ}\right)$. The spreading times of droplets under static releasing on these surfaces are $50 \mathrm{~min}, 2 \mathrm{~min}$, and $40 \mathrm{~s}$, respectively. For these surfaces, droplets were found to bounce off upon impacting on them and the lower limit of the impact velocity is plotted in Fig. 5. It is seen that it linearly depends on the shear modulus of the surface. This is consistent with the explanation of the air film. In Ref. [20], the qualitative analysis about the bounce of silicone oil droplets on the same fluid, which is vibrating, is performed. The critical acceleration for the rebound in our case is consistent with the prediction in Ref. [20] in the limit of low frequency.

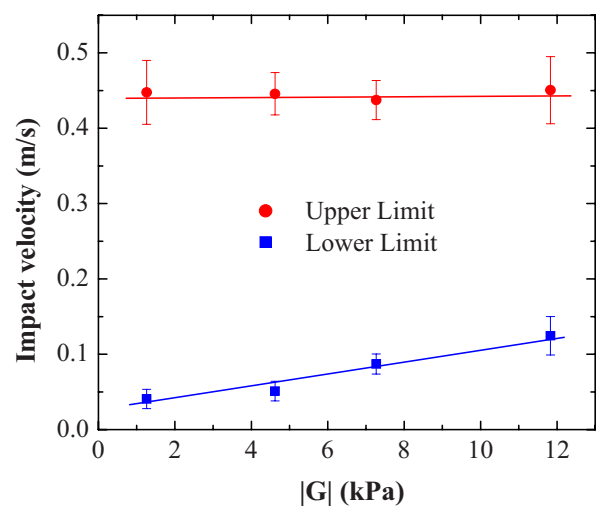

FIG. 5. (Color online) The lower and upper impact velocity limits for droplets impinging on surfaces of different shear moduli.

After the droplet contacts the surface, the droplet deforms and spreads due to the water hammer and dynamic pressures. When the Weber number $\mathrm{We}=\rho V^{2} R / \gamma>1(V>0.25 \mathrm{~m} / \mathrm{s}$ in this work), a cavity at the center of the droplet is formed and the droplet takes a toroidal shape in the spreading stage, which is shown in Fig. 6(a) for different impact velocities. If the impact height is sufficiently large, the cavity reaches deep into the droplet. When the droplet recoils, the top of the cavity retracts faster than the bottom and the air in the cavity is entrapped in the droplet, which forms a bubble. This case

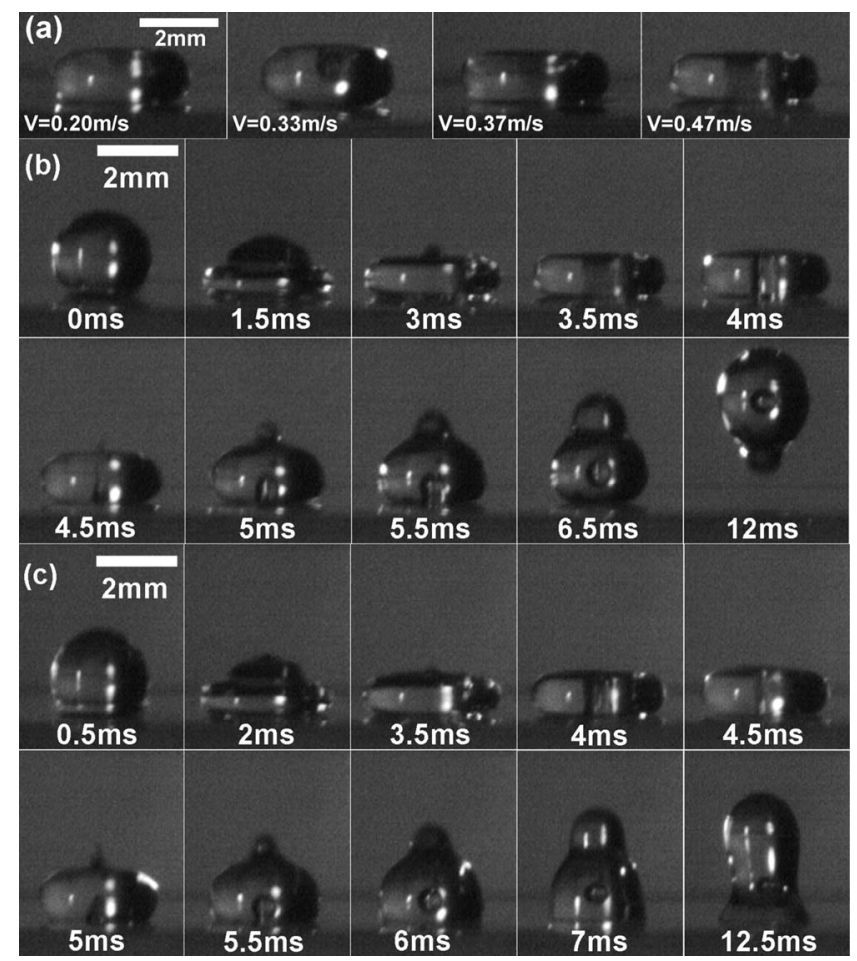

FIG. 6. (a) Air cavity and toroidal shape of droplets under different impact velocities. (b) The formation of air bubble entrapped from the cavity with $V=0.47 \mathrm{~m} / \mathrm{s}$. The bubble does not hit the air film beneath the droplet and the surface still exhibits superhydrophobicity. (c) The air bubble coalesces with the air film, which is released afterward and the surface becomes wetting when $V=0.49 \mathrm{~m} / \mathrm{s}$. 


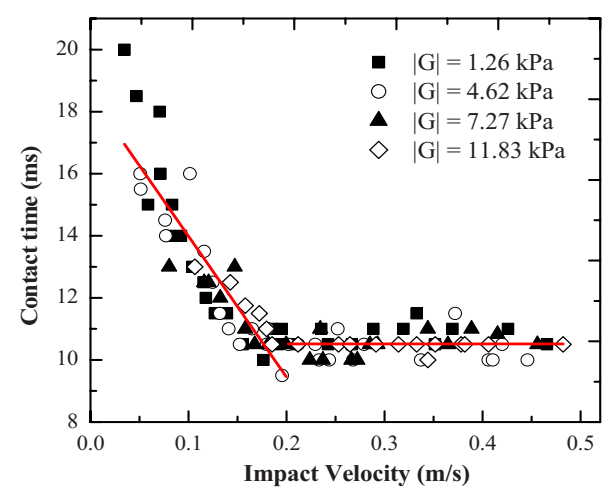

FIG. 7. (Color online) The contact time for different surfaces as a function of impact velocity.

was found when the impact velocity reached $0.47 \mathrm{~m} / \mathrm{s}$, as shown in Fig. 6(b), where the surface was still nonwetting.

The air bubble entrapped from the cavity can be a disaster to the rebound of the droplet because the air bubble can hit and coalesce with the air film beneath the droplet. Once the air film is released, the surface energy becomes large and consequently the droplet will wet and cannot bounce off the surface. This happened when the impact velocity reached $0.49 \mathrm{~m} / \mathrm{s}$ [Fig. 6(c)]. Our facilities limit us to the observation of the coalescence between the air bubble and film. However, based on the role of the air film discussed in Ref. [20], the shapes of the droplets in Figs. 6(b) and 6(c) might reveal the release of the air film. In Fig. 6(b), the ACA at $6.5 \mathrm{~ms}$ is equal to $150^{\circ}$, which shows that the surface is superhydrophobic and the air film should remain in place. However, in Fig. 6(c), the ACA at $6 \mathrm{~ms}$ is about $101^{\circ}$, indicating that the surface energy becomes large, which is caused by the detach of the air film.
The release of the air film by the air bubble entrapped from the cavity determines the upper limit of the impact velocity, above which the high dynamic pressure generates a deep cavity and causes the coalescence to take place. Therefore, this upper velocity limit is largely dependent on the impact height. This is confirmed by the upper limit of the impact velocity in Fig. 5, which remains almost constant for different surfaces.

For droplet-surface interactions, the contact time is an important parameter, which is defined as the time that a droplet is in contact with the surface before bouncing off [12]. The contact times for all the surfaces mentioned previously are plotted in Fig. 7. It is seen that the contact time decreases linearly with the impact velocity for $V<0.2 \mathrm{~m} / \mathrm{s}$, after which the contact time remains almost constant. This is consistent with the previous work [28], where the theoretical predictions and experimental results are reported.

In summary, we have shown that water droplets could bounce off nonsuperhydrophobic surfaces in a range of impact velocities. The rebound is caused by an air film beneath the droplet, which reduces the surface energy. The lower limit of the impact velocity is determined by the formation of the air film, while the upper limit is governed by the dynamic pressure, which, if sufficiently high, can cause the coalescence of the air bubble entrapped from the cavity and the air film beneath the droplet.

The authors thank the Impact Dynamics Laboratory at HKUST for the high-speed charge-coupled device camera and Smart Materials Laboratory for the rheometer. This work was supported in part by the Direct Allocation Grant No. DAG07/08.EG02 and School Initiative Grant No. SBI07/ 08.EG01 of HKUST.
[1] A. Marmur, Langmuir 20, 3517 (2004).

[2] J. B. Boreyko and C. H. Chen, Phys. Rev. Lett. 103, 174502 (2009).

[3] T. Deng, K. K. Varanasi, M. Hsu, N. Bhate, C. Keimel, J. Stein, and M. Blohm, Appl. Phys. Lett. 94, 133109 (2009).

[4] D. Oner and T. J. McCarthy, Langmuir 16, 7777 (2000).

[5] T. Baldacchini, J. E. Carey, M. Zhou, and E. Mazur, Langmuir 22, 4917 (2006).

[6] H. Tavana, A. Amirfazli, and A. W. Neumann, Langmuir 22, 5556 (2006).

[7] L. Feng, S. H. Li, Y. S. Li, H. J. Li, L. J. Zhang, J. Zhai, Y. L. Song, B. Q. Liu, L. Jiang, and D. B. Zhu, Adv. Mater. 14, 1857 (2002).

[8] M. Miwa, A. Nakajima, A. Fujishima, K, Hashimoto, and T. Watanabe, Langmuir 16, 5754 (2000).

[9] Y. Xiu, L. Zhu, D. W. Hess, and C. P. Wong, Nano Lett. 7, 3388 (2007).

[10] J.-Y. Shiu, C.-W. Kuo, P. Chen, and C.-Y. Mou, Chem. Mater. 16, 561 (2004).

[11] A. Tuteja, W. Choi, J. M. Mabry, G. H. Mckinley, and R. E. Cohen, Proc. Natl. Acad. Sci. U.S.A. 105, 18200 (2008).

[12] D. Richard, C. Clanet, and D. Quéré, Nature (London) 417,
811 (2002).

[13] K. K. S. Lau, J. Bico, K. B. K. Teo, M. Chhowalla, and G. A. J. Amaratunga, Nano Lett. 3, 1701 (2003).

[14] Y. C. Hong and H. S. Uhm, Appl. Phys. Lett. 88, 244101 (2006).

[15] M. Barberoglou, V. Zorba, E. Stratakis, E. Spanakis, P. Tzanetakis, S. H. Anastasiadis, and C. Fotakis, Appl. Surf. Sci. 255, 5425 (2009).

[16] Z. Wang, C. Lopez, A. Hirsa, and N. Koratkar, Appl. Phys. Lett. 91, 023105 (2007).

[17] Y. C. Jung and B. Bhushan, Langmuir 24, 6262 (2008).

[18] R. Kannan and D. Sivakumar, Colloids Surf., A 317, 694 (2008).

[19] Y. Couder, S. Protière, E. Fort, and A. Boudaoud, Nature (London) 437, 208 (2005).

[20] Y. Couder, E. Fort, C.-H. Gautier, and A. Boudaoud, Phys. Rev. Lett. 94, 177801 (2005).

[21] G. R. Lester, J. Colloid Sci. 16, 315 (1961).

[22] M. E. R. Shanahan and P. G. de Gennes, in Adhesion 11, edited by K. W. Allen (Elsevier Applied Science, London, 1987).

[23] K. K. Haller, D. Poulikakos, Y. Evntikos, and P. Monkewitz, J. Fluid Mech. 490, 1 (2003). 
[24] J. E. Field, Phys. Med. Biol. 36, 1475 (1991).

[25] V. Mehdi-Nejad, J. Mostaghimi, and S. Chandra, Phys. Fluids 15, 173 (2003).

[26] S. Chandra and C. T. Avedisian, Proc. R. Soc. London, Ser. A 432, 13 (1991).
[27] R. D. Schroll, C. Josserand, S. Zaleski, and W. W. Zhang, Phys. Rev. Lett. 104, 034504 (2010).

[28] K. Okumura, F. Chevy, D. Richard, D. Quéré, and C. Clanet, EPL 62, 237 (2003). 